\title{
Autonomic Response Evaluation during Gradual Body Weight Support: Comparison between Spectral and Symbolic Analysis
}

\author{
V Magagnin ${ }^{1,2}$, EG Caiani ${ }^{1,2}$, L Fusini ${ }^{1}$, M Turiel $^{2,3}$, \\ S Cerutti ${ }^{1}$, A Porta ${ }^{2,3}$ \\ ${ }^{1}$ Politecnico di Milano, Biomedical Engineering Dpt, Milan, Italy \\ ${ }^{2}$ Istituto Ortopedico IRCCS "R Galeazzi", Milan, Italy \\ ${ }^{3}$ Università degli Studi di Milano, Dpt of Technologies for Health, Milan, Italy
}

\begin{abstract}
The robot-driven walking therapies with body weight support (BWS) are recently gaining appreciation as they can improve gait recovery in stroke survivors. However, $B W S$ could evoke a response of the autonomic nervous system (ANS) that could be dangerous in patients with cardiovascular regulation deficit. The aim of this study is to evaluate the ANS response during BWS phases through spectral and symbolic analyses of short-term heart rate variability. This evaluation could help to tailor robotdriven walking strategies preventing discomfort and improving rehabilitation. A group of 10 normal subjects was studied during several BWS phases. Results from spectral and symbolic analyses indicated a gradual sympathetic activation together with a parasympathetic inhibition with increasing percentages of BWS. However, since changes appear mainly as trends, our findings suggest that the progressive increase of the BWS generates a light sympathetic stimulation.
\end{abstract}

\section{Introduction}

Treadmill training with body weight support (BWS) has been recently proposed as a clinical tool helpful to restore gait in individuals with partial loss of motor skills [1]-[4].

In BWS, the body weight is partially unloaded through a harness-counterweight system, in order to delay muscle fatigue, and to allow for locomotion in uncompromised mechanical form until the lower extremities can gain the strength, endurance, and coordination necessary for independent locomotion during full weight bearing [5].

We hypothesized that the experimental protocol for BWS with robotic-assisted locomotion could generate alterations on the cardiovascular system due to the experimental procedure itself. In fact, the process of instrumentation (Figure 1) requires to suspend the subject for several minutes ( 5 to $10 \mathrm{~min}$ ). This represents a nonphysiological condition that could evoke a response of the autonomic nervous system that could be particularly dangerous in patients with cardiovascular deficit, more prone to orthostatic intolerance, or in patients usually sitting in a wheelchair for long periods of their day. The analysis of the power spectrum density of the short-term fluctuations of heart beat durations (RR-interval), derived from ECG tracings, represents an indirect and noninvasive method to assess changes in autonomic function.

By this method, the spectral low frequency components (LF: from 0.04 to $0.15 \mathrm{~Hz}$ ) and the spectral

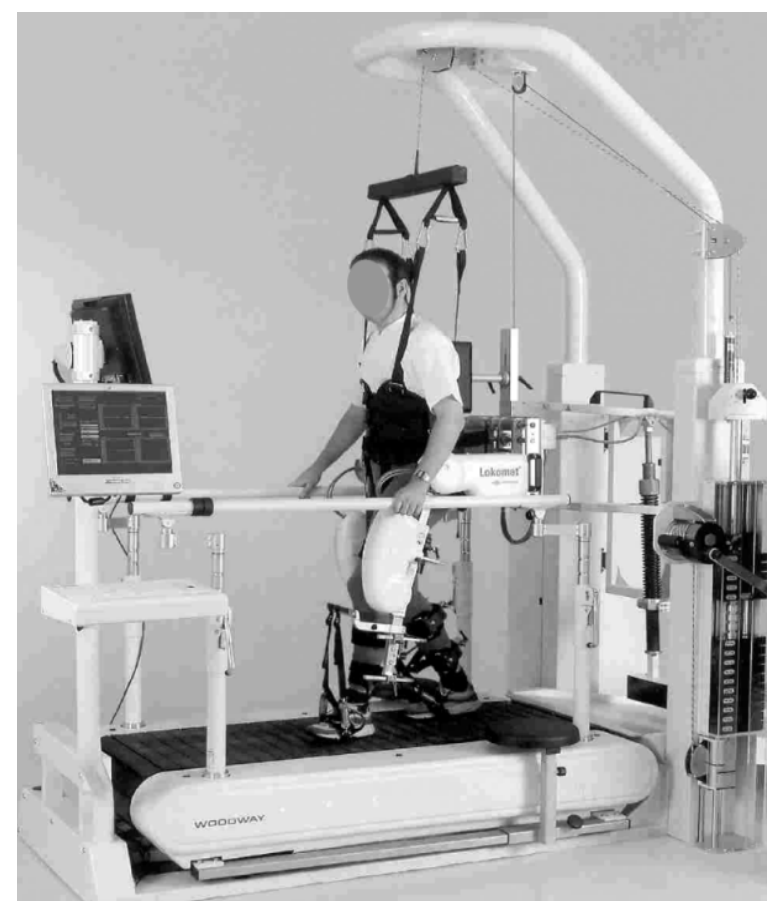

Figure 1. A participant ambulating on a treadmill with assistance from the Lokomat (www.hocoma.ch) 
high frequency components (HF: from 0.15 to $0.4 \mathrm{~Hz}$ ), expression of sympathetic and vagal modulations [6], can be inferred and quantified.

Symbolic analysis of the short-term fluctuations of heart beat duration, is a recently proposed technique able to detect non reciprocal subtle changes in sympathetic and parasympathetic modulations or reciprocal changes with different magnitudes [7].

Our goal was to evaluate the autonomic nervous system response during BWS in order to help to tailor robot-driven walking strategies preventing cardiovascular discomfort and improving rehabilitation. Accordingly, we studied in 10 normal subjects the ECG and respiratory fluctuations during different body weight unloading.

\section{Methods}

\subsection{Experimental protocol}

We studied 10 normal volunteers (mean age, $23 \pm 2.3$ years) undergoing 5 different BWS phases obtained via robot-driven gait orthosis, Lokomat (Hocoma, Volketswil, Switzerland.

Each subject was monitored for a total of 45 minutes, during the following experimental conditions:

1) S_0: 5 minutes of recording during standing position with $0 \%$ of BWS;

2) S_25: as for S_0 with $25 \%$ of BWS;

3) S 50: as for S 0 with $50 \%$ of BWS;

4) S_75: as for S_0 with 75\% of BWS;

5) S_100: 5 minutes during the suspended phase (100\% of BWS).

The BWS percentages were applied in random order and followed each one by 5 minutes of rest in sitting position.

For each subject, the continuous I-lead ECG signal was acquired (sampling frequency $2048 \mathrm{~Hz}$ ), by positioning the electrodes in correspondence to the right and left collarbones, and to the C5 cervical vertebra. This electrode configuration has been applied due to the fact that the abdominal body harness, necessary for the BWS phases, did not allow the conventional third electrode placement in the lower part of the thorax. Moreover, the respiratory signal was also recorded (sampling frequency $256 \mathrm{~Hz}$ ) with a piezoelectric thoracic belt, positioned at abdominal level below the abdominal body harness.

The ECG and respiratory transducers were connected to a multi-modality device for real-time computerized biofeedback and data acquisition (ProComp Infiniti ${ }^{\mathrm{TM}}$ encoder, Thought Technology Ltd., Montreal, Canada).

\subsection{Signal analysis}

The ECG signal was analyzed with custom software developed using Matlab (The Mathworks, Inc.; Natick,
Massachusetts) in order to detect the $\mathrm{R}$ peaks and calculate the RR variability series as the time interval between two consecutive $\mathrm{R}$ peaks, detected by a traditional derivative threshold procedure. The respiratory signal was oversampled to $2048 \mathrm{~Hz}$ in order to obtain temporal correspondence with the ECG. Then, the respiratory signal was sampled in correspondence to the temporal position of the detected $\mathrm{R}$ waves, in order to extract the respiratory beat to beat series. Variability series of approximately 300 beats for each experimental condition were analyzed.

Spectral analysis was performed by means of autoregressive spectral methods with software developed in-house. The Levinson-Durbin recursion was used to identify the coefficients of the autoregressive model [8] and the order was chosen (between 4 and 12) accordingly to the Akaike figure of merit. The autoregressive spectral decomposition procedure was applied to calculate the power of the oscillations embedded in the series [9]. The rhythms were classified [10] as very low frequency (VLF, $<0.04 \mathrm{~Hz}$ ), low-frequency (LF, ranging from 0.04 to $0.14 \mathrm{~Hz})$ and high frequency $(\mathrm{HF}, \pm 0.03 \mathrm{~Hz}$ around the respiratory frequency detected on the respiratory signal) oscillations. The whole process of automatic extraction of beat-to-beat series and their spectral analysis took a trained clinician approximately 5 minutes for each examined subject. In tables, the power is expressed in absolute unit $\left(\mathrm{LF}_{\mathrm{RR}}\right.$ and $\left.\mathrm{HF}_{\mathrm{RR}}\right)$, in normalized unit (LFnu and HFnu, as $100 * \mathrm{LF}_{\mathrm{RR}} /$ (Variance-VLF) and $100 * \mathrm{HF}_{\mathrm{RR}} /$ (Variance-VLF), respectively) and in percentage of the total power $\left(\mathrm{LF}_{\mathrm{RR}} \%\right.$ and $\mathrm{HF}_{\mathrm{RR}} \%, 100 *$ $\mathrm{LF}_{\mathrm{RR}} /$ Variance and $100 * \mathrm{HF}_{\mathrm{RR}} /$ Variance, respectively). Respiratory spectrum was used to assess the main respiratory frequency $\left(\mathrm{RESP}_{\text {freq }}\right)$. The power at $\mathrm{RESP}_{\text {freq }}$ on the respiratory signal $\left(\mathrm{RESP}_{\text {power }}\right)$ in each experimental condition was divided by the same index calculated at S_0 (dRESP\% $\left.\%_{\text {power }}\right)$.

Symbolic analysis was carried out as proposed by Porta et al. [7]. Briefly, the series were uniformly quantized over 6 quantization bins, thus becoming a series of integer values (symbols) ranging from 0 to 5 . Symbols were grouped into sequences (words) of three symbols. These words were classified into 4 families according to the type and number of variations between two consecutive symbols: i) no variation $(0 \mathrm{~V})$; ii) one variation $(1 \mathrm{~V})$; iii) two like variations $(2 \mathrm{LV})$ i.e. two variations with the same sign; iv) two unlike variations (2UV) i.e. two variations with opposite sign. The rates of occurrence of these families defined as $0 \mathrm{~V} \%, 1 \mathrm{~V} \%$, $2 \mathrm{LV} \%$, and $2 \mathrm{UV} \%$, were evaluated by dividing the number of times that a pattern belonging to a specific family by N-2 (multiplied by 100). 
Table 1: Spectral and Symbolic parameters during: 0\% BWS (S_0), 25\% BWS (S_25), 50\% (S_50), 75\% BWS (S_75), $100 \%$ BWS (S_100)

\begin{tabular}{lcccccc}
\hline \hline & & \multicolumn{5}{c}{ BWS PHASES } \\
\cline { 3 - 7 } & & $\mathrm{S} \_0$ & $\mathrm{~S} \_25$ & $\mathrm{~S}$ 50 & $\mathrm{S}$ 75 & S_100 \\
\hline $\mathrm{RR}$ & $(\mathrm{ms})$ & $789(689-806)$ & $787(708-804)$ & $779(710-806)$ & $758(700-779) *$ & $747(671-793) *$ \\
Variance & $\left(\mathrm{ms}^{2}\right)$ & $3025(1811-4347)$ & $2532(1284-4153)$ & $2366(1789-3772)$ & $2516(1909-2591)$ & $2803(877-3678)$ \\
$\mathrm{LF}_{\text {power }}$ & $\left(\mathrm{ms}^{2}\right)$ & $942(758-1739)$ & $917(372-1751)$ & $815(430-1497)$ & $1072(754-1269)$ & $516(426-1173)$ \\
$\mathrm{HF}_{\text {power }}$ & $\left(\mathrm{ms}^{2}\right)$ & $87(29-174)$ & $46(33-258)$ & $35(27-223)$ & $43(26-187)$ & $47(9-144) *$ \\
$\mathrm{LF}_{\text {nu }}$ & $(\mathrm{nu})$ & $88.5(69.5-93.8)$ & $92.0(75.4-101.8)$ & $90.1(80.4-94.7)$ & $90.2(77.1-93.6)$ & $90.8(79.2-94.7)$ \\
$\mathrm{HF}_{\text {nu }}$ & $(\mathrm{nu})$ & $3.8(2.4-18.1)$ & $4.1(2.2-17.3)$ & $4.8(4.4-8.2)$ & $4.6(25-12.9)$ & $2.4(1.0-4.92)$ \\
$\mathrm{LF}_{\mathrm{RR} \%}$ & $(\%)$ & $53.0(36.9-62.4)$ & $47.5(35.7-61.5)$ & $35.8(25.8-58.0)$ & $51.2(36.6-61.7)$ & $53.2(32.5-65.9)$ \\
$\mathrm{HF} F_{\mathrm{RR}} \%$ & $(\%)$ & $2.3(1.4-9.8)$ & $2.8(1.2-8.4)$ & $2.3(1.4-4.8)$ & $3.6(1.7-7.2)$ & $2.1(0.8-6.1)$ \\
$\mathrm{RESP}_{\text {freq }}$ & $(\mathrm{Hz})$ & $.28(.20-.33)$ & $.28(.24-.30)$ & $.24(.18-.27)$ & $.26(.23-.32)$ & $.33(.32-.36) *$ \\
$\mathrm{dRESP}_{\text {power }}$ & $(--)$ & 1 & $.32(.18-.65) *$ & $.65(.25-.88) *$ & $.62(.52-.93) *$ & $.26(.22-.59) *$ \\
\hline $0 \mathrm{~V} \%$ & & $42.3(33.7-46.5)$ & $37.2(36.2-44.6)$ & $38.9(34.9-45.9)$ & $40.9(24.0-49.5)$ & $37.6(32.7-49.7)$ \\
$1 \mathrm{~V} \%$ & & $45.5(43.2-48.3)$ & $47.6(45.1-49.1)$ & $48.6(41.7-49.3)$ & $42.9(39.2-49.0)$ & $46.1(37.5-51.7)$ \\
$2 \mathrm{LV} \%$ & & $8.1(5.1-9.6)$ & $7.0(5.3-9.8)$ & $8.0(4.8-9.7)$ & $7.4(6.5-12.9)$ & $5.1(4.1-8.5)$ \\
$2 \mathrm{UV} \%$ & & $5.4(3.9-5.8)$ & $6.4(6.1-7.4)$ & $5.6(5.1-7.2)$ & $6.3(5.3-9.4)$ & $7.5(6.4-9.0)$ \\
\hline \hline
\end{tabular}

*:p $<0.05$ paired t-test with Bonferroni correction v S_0

\subsection{Statistical analysis}

For both spectral and symbolic analysis, Friedman test $(\mathrm{p}<.05)$ was used to evaluate the global differences in the Bonferroni correction was applied to test the changes in the parameters at each experimental step compared to their baseline values.

\section{Results}

\subsection{Spectral analysis}

RR mean duration was characterized by a non significant decreasing trend with increasing percentage of BWS from S_0 to S_50. Moreover during S_75 and S_100 significant reductions of respectively 31 and $42 \mathrm{~ms}$ were noticed, compared to S_0. Significant changes were found in the RR HF band: indeed HF power expressed in absolute units decreased, in particular during S_100 compared to S_0 $\left(40 \mathrm{~ms}^{2}\right)$. The LFnu and HFnu powers showed respectively a non significant increasing and decreasing trend with increasing percentages of BWS.

The Friedman test showed a significant change in respiratory frequency $\mathrm{RESP}_{\text {freq }}$ during the experimental protocol. The power at the RESP freq $_{\text {normalized by that at }}$ S_0, dRESP\% $\%_{\text {power }}$, showed a significant decrease during all the experimental phases, compared to S_0.

\subsection{Symbolic analysis}

The symbolic indexes did not show any significant variation during the different phases of the experimental protocol. However, an increasing trend during $75 \%$ and $100 \%$ BWS was evidenced in $2 \mathrm{UV} \%$, together with a corresponding decrease in $0 \mathrm{~V} \%$.

\section{Discussion and conclusions}

In our protocol, we analyzed 10 volunteers, to evaluate the effects of the application of different BWS percentages on the cardiac autonomic nervous system activity via spectral and symbolic analyses of heart rate variability series.

Results from both autoregressive spectral analysis and symbolic analysis indicate a gradual sympathetic activation together with a parasympathetic inhibition with increasing percentages of BWS. However, since changes are not remarkable and appear mainly as trends, our findings suggest that the progressive increase of the BWS generates a light cardiovascular response compared to stand in healthy subjects. Further analysis is necessary to understand whether in pathological subjects with cardiovascular regulation deficit, this partial BWS is viable.

Our results could help to tailor robot-driven walking therapies, preventing discomfort and improving rehabilitation. Moreover they could provide a reference values for any comparison with pathological populations.

\section{References}

[1] Dobkin BH, Harkema S, Requejo P,. Edgerton VR. Modulation of locomotor-like EMG activity in subjects with complete and incomplete spinal cord injury. J Neurol 
Rehabil 1995; 9 (5): 183-190.

[2] Wernig A, Muller S. Laufband locomotion with body weight support improved walking in persons with severe spinal cord injuries. Paraplegia 1992; 30 (4): 229-238.

[3] Wernig A, Muller S, Nanassy A, Cagol E. Laufband therapy based on 'rules of spinal locomotion' is effective in spinal cord injured persons. Eur J Neurosci 1995; 7: 823-829.

[4] Wernig A, Nanassy A, Muller S. Laufband (treadmill) therapy in incomplete paraplegia and tetraplegia. J Neurotrauma 1999; 16: 719-726.

[5] Nash MS, Jacobs PL, Johnson BM, Field-Fote E. Metabolic and cardiac responses to robotic-assisted locomotion in motor-complete tetraplegia: a case report. J Spinal Cord Med 2004; 27: 78-82.

[6] Malik M,. Camm AJ, Bigger JT, Beithard G, et al. Heart rate variability. Standard of measurement, physiological interpretation, and clinical use. Eur Heart J 1996; 17 (3): 354-381.

[7] Porta A, Tobaldini E, Guzzetti S, Furlan R, Montano N, Gnecchi-Ruscone T. Assessment of cardiac autonomic modulation during graded head-up tilt by symbolic analysis of heart rate variability. Am J Physiol 2007; 293: H702-H708

[8] Kay SM. Modern Spectral Analysis: Theory and Applications. Ed. Prentice Hall (Englewood Cliffs, NJ) 1988

[9] Zetterberg LH. Estimation of parameters for a linear difference equation with application to EEG analysis. Math Biosci 1979: 227-75

[10] Task Force of the European Society of Cardiology and North American Society of Pacing and Electrophysiology: "Heart rate variability, standard of measurement, physiological interpretation and clinical use". Circulation 1996; 93: 1043-65

Address for correspondence

Enrico G Caiani, PhD

Dipartimento di Bioingegneria, Politecnico di Milano

Via Golgi 39, 20133, Milano, Italy

enrico.caiani@biomed.polimi.it 